\title{
Diastereoselective synthesis of phosphonato esters by reaction between triphenylphosphite and acetylenic esters in the presence of NH-acid compounds
}

\author{
Malek T. Maghsoodlou ${ }^{a^{*},}$ Nourollah Hazeri ${ }^{a}$, Sayyed M. Habibi-Khorassani ${ }^{\text {a }}$, Lotfali \\ Saghatforoush $^{\text {b }}$, Mohammad K. Rofouei, ${ }^{c}$ and Mojtaba Rezaie ${ }^{a}$ \\ a Department of Chemistry, The University of Sistan and Balouchestan, P. O. Box 98135-674, \\ Zahedan, Iran \\ b Faculty of Science, Payame Nour University of Khoy, Khoy, Iran \\ c Faculty of Chemistry, The University of Tarbiat Moallem, Tehran, Iran \\ * Corresponding author: +98-541-2446565; fax: +98-541-2446565; \\ E-mail: MT maghsoodlou@yahoo.com
}

\begin{abstract}
The reaction of dialkyl acetylenedicarboxylates with $\mathrm{N}-\mathrm{H}$ acids such as phthalimide and saccharin in the presence of triphenyl phosphite at room temperature led to stable phosphonato ester derivatives 3a-d. With respect to the Karplus equation and coupling constants, the configurations of compounds $\mathbf{3 a}$-d were determined ( $2 \mathrm{R}^{*}, 3 \mathrm{~S}^{*}$ and mirror image).
\end{abstract}

Keywords: Phthalimide and saccharin, triphenylphosphite, Karplus equation, diastereoselective synthesis, phosphonato esters

\section{Introduction}

In recent years there has been increasing interest in the synthesis of organophosphorus compounds, in particular those bearing a carbon atom bound to a phosphorus atom. This interest has resulted from the recognition of the value of such compounds in a wide range of industrial, biological and chemical synthetic aspects. ${ }^{1,2}$

New or improved methods for phosphonate synthesis continue to attract much attention because phosphonates present biologically important properties and serve as natural products, analogues of phosphates, phosphonopeptides, aminoacid analogues and pro drugs. It appears that they have an important role in the therapeutic behavior of anti cancer and anti-HIV drugs. Given their ubiquitous use as pharmacological agents and crucial synthetic intermediates and reagents (e.g. Horner-Wadsworth-Emmons and related reactions), construction of the P-C bond still remains a formidable challenge. ${ }^{3-5}$ 
Traditionally, organophosphonates are prepared via a Michaelis-Arbuzov or a MichaelisBecker reaction utilizing the nucleophilic properties of trivalent phosphorus compounds (e.g. trialkyl phosphites or alkali metal salts of dialkyl phosphates) in the presence of alkyl halides.

Depending on the methods of choice, these conventional reaction conditions often are not convenient, requiring elevated temperature, the use of a strong anhydrous base, and very long reaction times. Moreover, these procedures often lead to a complicated mixture of side products or result in poor yields of the phosphonate. Therefore, to circumvent these aforementioned chronic problems, numerous other methods have been investigated. Nevertheless, each of these methods lacks in generality, prompting us to embark on a milder and improved procedure better suited toward the synthesis of phosphonato esters. ${ }^{3-10}$

\section{Results and Discussion}

In this work, we wish to report a simple, rapid, neutral, room temperature and stereoselective synthesis of phosphonate esters from the reaction between triphenylphosphite and acetylenic esters in the presence of N-H acids, such as phthalimide or saccharin (see Scheme $1)$.

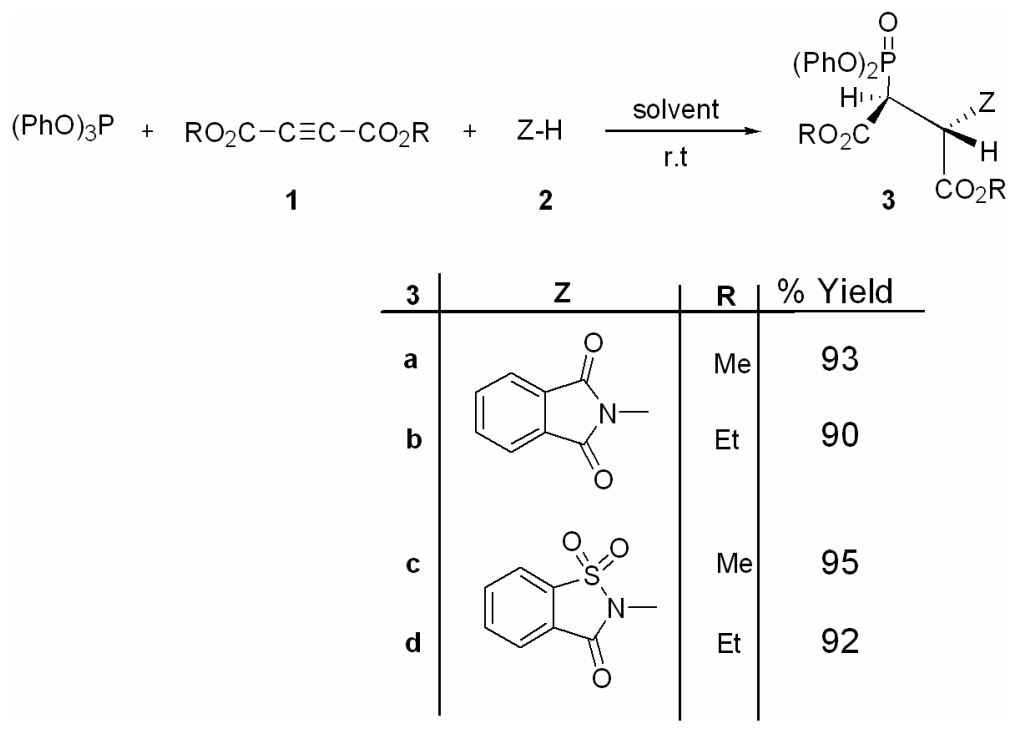

\section{Scheme 1}

The essential structure of the products was deduced from their mass spectra, ${ }^{13} \mathrm{C},{ }^{1} \mathrm{H},{ }^{31} \mathrm{P}$ NMR and IR spectral data. The ${ }^{1} \mathrm{H}$ NMR spectrum of 3a-d displayed two signals for vicinal methane protons which appear as double doublets. The vicinal proton-proton coupling constant $\left({ }^{3} \mathrm{~J}_{\mathrm{HH}}\right)$ can help, as a function of the torsion angle, to determine the proton position by the Karplus equation. ${ }^{11}$ The presence of ${ }^{31} \mathrm{P}$ in 3a-d helps in the assignment of the signals by long 
range coupling with ${ }^{1} \mathrm{H}$ and ${ }^{13} \mathrm{C}$ nuclei. The comparison of observed ${ }^{3} J_{\mathrm{CP}}$ for the $\mathrm{C}=\mathrm{O}$ group with the desired values indicates the product geometries. It is found that, there is only one of the two probable diastereoisomers, in the hydrolysis products.

The work reported here was undertaken in order to determine the possibility of trapping the reactive 1:1 intermediate formed in the initial reaction between triphenyl phosphite and dimethyl acetylenedicarboxylate (DMAD) by a strong $\mathrm{N}-\mathrm{H}$ acid such as phthalimide. This three component reaction produces phosphonate ester $\mathbf{3}$ which apparently results from initial addition of the triphenyl phosphite to the acetylenic ester and concomitant protonation of the 1:1 adduct, then attack by the anion to form intermediate $\mathbf{A}$, which is convert to the phosphonato ester $\mathbf{3}$ by nucleophilic attack on the phosphorus group by water (see Scheme 2).

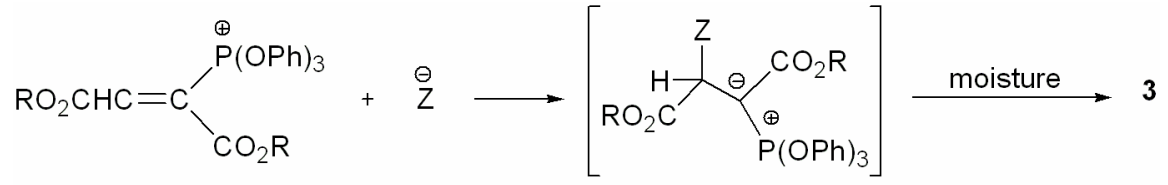

A

\section{Scheme 2}

Selected ${ }^{1} \mathrm{H},{ }^{13} \mathrm{C}$, and ${ }^{31} \mathrm{P}$ NMR chemical shifts ( $\delta$ in ppm) and coupling constants $(\mathrm{J}$ in $\mathrm{Hz})$ for $\mathrm{H}-2, \mathrm{H}-3, \mathrm{CO}_{2} \mathrm{R}, \mathrm{C}-2$, and $\mathrm{C}-3$ with respect to scheme 3 in compounds 3a-d have been reported in Table 1 (see Scheme 2 and Table 1).

Table 1. Selected data and features

\begin{tabular}{|c|c|c|c|c|c|c|c|c|c|c|c|c|c|}
\hline \multirow{2}{*}{ Compound } & \multicolumn{4}{|c|}{${ }^{1} \mathrm{H}$ NMR } & \multicolumn{1}{|c|}{${ }^{13} \mathrm{C} \mathrm{NM}$} & \\
\cline { 2 - 14 } & $\delta \mathrm{H}_{3}$ & ${ }^{2} J_{\mathrm{HP}}$ & $\delta \mathrm{H}_{2}$ & ${ }^{3} J_{\mathrm{HP}}$ & $\delta \mathrm{C}_{4}$ & ${ }^{2} J_{\mathrm{CP}}$ & $\delta \mathrm{C}_{3}$ & ${ }^{1} J_{\mathrm{CP}}$ & $\delta \mathrm{C}_{2}$ & ${ }^{2} J_{\mathrm{CP}}$ & $\delta \mathrm{C}_{1}$ & ${ }^{3} J_{\mathrm{CP}}$ & $\mathrm{NMR}$ \\
\hline 3a & 4.64 & 20.9 & 5.99 & 5.5 & 166.65 & 7.5 & 45.18 & 134.6 & 49.36 & 4.5 & 168.27 & 19.4 & 10.13 \\
\hline 3b & 4.57 & 20.8 & 5.92 & 5.9 & 166.08 & 7.4 & 45.34 & 134.6 & 49.45 & 4.6 & 167.77 & 18.7 & 10.43 \\
\hline 3c & 4.52 & 20.9 & 5.87 & 5.4 & 166.66 & 7.5 & 45.07 & 134.4 & 49.28 & 4.7 & 168.97 & 19.5 & 10.42 \\
\hline 3d & 4.58 & 20.9 & 5.93 & 5.4 & 166.09 & 7.3 & 45.27 & 133.7 & 49.40 & 4.6 & 167.02 & 19.4 & 10.73 \\
\hline
\end{tabular}

The vicinal proton-proton coupling constant $\left({ }^{3} J_{\mathrm{HH}}\right)$ as a function of the torsion angle can be obtained from the Karplus equation. ${ }^{11}$ Typically, $J_{\text {gauche }}$ varies between 1.5 and $5 \mathrm{~Hz}$ and $J_{\text {anti }}$ between 10 and $14 \mathrm{~Hz}$. Observation of ${ }^{3} J_{\mathrm{HH}}=11.5 \mathrm{~Hz}$ for the vicinal protons in compound 3 (see Experimental) indicates an anti arrangement for these protons. Since compound $\mathbf{3}$ possesses two chirality centers, two diastereoisomers with anti $\mathrm{H}-\mathrm{C}-\mathrm{C}-\mathrm{H}$ arrangements are possible (see Scheme 3). 


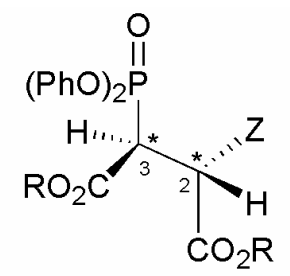

$\left(2 R^{*}, 3 S^{*}\right)-3$ or $\left(2 S^{*}, 3 R^{*}\right)-3$

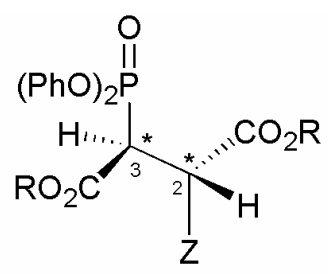

$\left(2 S^{*}, 3 S^{*}\right)-3$ or $\left(2 R^{*}, 3 R^{*}\right)-3$

\section{Scheme 3}

The three-bond carbon-phosphorus coupling ${ }^{3} J_{\mathrm{CP}}$, depends on configuration, as expected, transoid coupling being larger than cisoid. The Karplus relation can be derived from the data for organophosphorus compounds with tetra and penta-coordinate phosphorus. ${ }^{12}$ The ${ }^{3} J_{\mathrm{CP}}$ for the PC-C-C(O) moiety is $19.4,18.7,19.5$ and $19.4 \mathrm{~Hz}$ respectively which corresponds to $\left(2 \mathrm{R}^{*}, 3 \mathrm{~S}^{*}\right)-3$ and its mirror image $\left(2 \mathrm{~S}^{*}, 3 \mathrm{R}^{*}\right)$ geometries (Experimental section).

In conclusion a diastereoselective synthesis of phosphonates has been developed. Our strategy allows the preparation of functionalized phosphonato ester derivatives, which are otherwise difficult to make. Particularly attractive is the possibility of access to new phosphonato esters in diastereomerically pure form.

\section{Experimental Section}

General Procedures. Melting points and IR spectra of all compounds were measured on an Electrothermal 9100 apparatus and a Shimadzu IR-460 spectrometer respectively. Also, the ${ }^{1} \mathrm{H}$,

${ }^{13} \mathrm{C}$, and ${ }^{31} \mathrm{P}$ NMR spectra were obtained from a BRUKER DRX-500 AVANCE instrument with $\mathrm{CDCl}_{3}$ as solvent at $500.1,125.8$, and $202.4 \mathrm{MHz}$ respectively. In addition, the mass spectra were recorded on a Shimadzu GC/MS QP 1100 EX mass spectrometer operating at an ionization potential of $70 \mathrm{eV}$. Elemental analysis for $\mathrm{C}, \mathrm{H}$ and $\mathrm{N}$ were performed using a Heraeus CHN-ORapid analyzer. Dialkyl acetylenedicarboxylates, triphenylphosphite, phthalimide and saccharin were purchased from Fluka, (Buchs, Switzerland) and used without further purification.

General synthetic procedure (Exemplified by 3a)

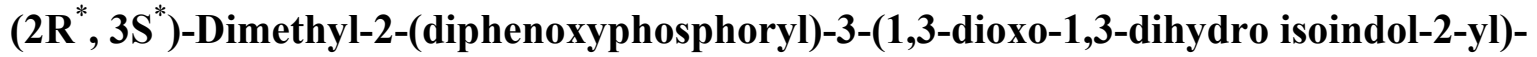
succinate (3a). To a magnetically stirred solution of triphenyl phosphite $(0.31 \mathrm{~g}, 1 \mathrm{mmol})$ and phthalimide $(0.137 \mathrm{~g}, 1 \mathrm{mmol})$ in ethyl acetate $(15 \mathrm{~mL})$ was added dropwise a dimethyl acetylene dicarboxylate $(0.142 \mathrm{~g}, 1 \mathrm{mmol})$. After approximately 24 hours stirring at room temperature, the solvent was removed under reduced pressure and the product washed with cold diethyl ether $(2 \times 5 \mathrm{~mL})$.

White powder, yield 93\%, mp=136-138 ${ }^{\circ} \mathrm{C}$. IR $(\mathrm{KBr})\left(v_{\max }, \mathrm{cm}^{-1}\right): 1766(\mathrm{C}=\mathrm{O}), 1718$ and 1738 $\left(\mathrm{CO}_{2} \mathrm{CH}_{3}\right), 1279(\mathrm{P}=\mathrm{O})$. MS, (m/z, \%): $429(\mathrm{M}-\mathrm{PhO}, 9), 283\left(\mathrm{M}-\mathrm{C}_{6} \mathrm{H}_{4} \mathrm{NO}_{2}\right.$ and $\left.\mathrm{OPh}, 30\right), 223$ (283- $\left.\mathrm{CH}_{3} \mathrm{COOH}, 84\right), 165$ (283-2 $\left.\mathrm{CO}_{2} \mathrm{CH}_{3}, 10\right), 94(\mathrm{OPh}, 9) .{ }^{31} \mathrm{P} \mathrm{NMR}\left(202.4 \mathrm{MHz}, \delta, \mathrm{CDCl}_{3}\right)$ : 
$10.13\left[\mathrm{~s},(\mathrm{PhO})_{2} \mathrm{P}(=\mathrm{O})\right] .{ }^{1} \mathrm{H}$ NMR $\left(500.1 \mathrm{MHz}, \delta, \mathrm{CDCl}_{3}\right): 3.77$ and $3.92\left(6 \mathrm{H}, 2 \mathrm{~s}, 2 \mathrm{CO}_{2} \mathrm{CH}_{3}\right)$, $4.64\left(1 \mathrm{H}, \mathrm{dd},{ }^{2} J_{\mathrm{PH}}=20.9 \mathrm{~Hz},{ }^{3} J_{\mathrm{HH}}=11.5 \mathrm{~Hz}, \mathrm{P}-\mathrm{CH}\right), 5.99\left(1 \mathrm{H}, \mathrm{dd},{ }^{3} J_{\mathrm{PH}}=5.5 \mathrm{~Hz},{ }^{3} J_{\mathrm{HH}}=11.5 \mathrm{~Hz}, \mathrm{P}-\right.$ $\mathrm{CH}-\mathrm{CH}), 7.05-7.90\left(14 \mathrm{H}, \mathrm{m}, \mathrm{H}_{\mathrm{Ar}}\right) .{ }^{13} \mathrm{C} \mathrm{NMR}\left(125.8 \mathrm{MHz}, \delta, \mathrm{CDCl}_{3}\right): 45.18\left(\mathrm{~d},{ }^{1} J_{\mathrm{CP}}=134.6 \mathrm{~Hz}\right.$, $\mathrm{P}-\mathrm{CH}), 49.36\left(\mathrm{~d},{ }^{2} J_{\mathrm{CP}}=4.5 \mathrm{~Hz}, \mathrm{P}-\mathrm{C}-\mathrm{CH}\right), 53.31$ and $53.57\left(\mathrm{~s}, 2 \mathrm{OCH}_{3}\right), 123.86,131.86,134.42$, 167.30 (s, C of $\mathrm{C}_{8} \mathrm{H}_{5} \mathrm{NO}_{2}$ ) 120.33, 120.46 (2d, $\mathrm{C}_{\text {ortho}}$ ), 125.47 and 125.56 (2d, $\mathrm{C}_{\text {para }}$ ), 129.73 (s, $\left.\mathrm{C}_{\text {meta }}\right), 149.59\left(\mathrm{~d},{ }^{2} \mathrm{~J}_{\mathrm{CP}}=9.8 \mathrm{~Hz}, \mathrm{C}_{\mathrm{ipso}}\right), 150.05\left(\mathrm{~d},{ }^{2} J_{\mathrm{CP}}=8.6 \mathrm{~Hz}, \mathrm{C}_{\mathrm{ipso}}\right), 166.65\left(\mathrm{~d},{ }^{2} J_{\mathrm{CP}}=7.5 \mathrm{~Hz}, \mathrm{P}-\mathrm{C}-\right.$ $\mathrm{C}=\mathrm{O}), 168.27\left(\mathrm{~d},{ }^{3} J_{\mathrm{CP}}=19.4 \mathrm{~Hz}, \mathrm{P}-\mathrm{C}-\mathrm{C}-\mathrm{C}=\mathrm{O}\right)$. Anal. Calcd for $\mathrm{C}_{26} \mathrm{H}_{22} \mathrm{NO}_{9} \mathrm{P}$ (523): C, 59.66; H, $4.20 ;$ N, 2.67\%, Found: C, 60.29; H, 4.15; N, 2.76\%.

(2R* $\left.\quad 3 S^{*}\right)$-Diethyl-2-(diphenoxyphosphoryl)-3-(1,3-dioxo-1,3-dihydro isoindol-2-yl)succinate (3b). White powder, yield 90\%, mp=104-106 ${ }^{\circ} \mathrm{C} . \mathrm{IR}(\mathrm{KBr})\left(v_{\max }, \mathrm{cm}^{-1}\right): 1771(\mathrm{C}=\mathrm{O})$, 1712 and $1733\left(\mathrm{CO}_{2} \mathrm{CH}_{2} \mathrm{CH}_{3}\right), 1285(\mathrm{P}=\mathrm{O}) .{ }^{31} \mathrm{P} \mathrm{NMR}\left(202.4 \mathrm{MHz}, \delta, \mathrm{CDCl}_{3}\right)$ : 10.43 [s, $\left.(\mathrm{PhO})_{2} \mathrm{P}(=\mathrm{O})\right]$. ${ }^{1} \mathrm{H}$ NMR $\left(500.1 \mathrm{MHz}, \delta, \mathrm{CDCl}_{3}\right): 1.19$ and $1.33\left(6 \mathrm{H}, 2 \mathrm{t}, \mathrm{CH}_{3}\right), 4.21$ and $4.34(4 \mathrm{H}$, $\left.2 \mathrm{q}, \mathrm{OCH}_{2}\right), 4.57\left(1 \mathrm{H}, \mathrm{dd},{ }^{2} J_{\mathrm{PH}}=20.8 \mathrm{~Hz},{ }^{3} J_{\mathrm{HH}}=11.5 \mathrm{~Hz}, \mathrm{P}-\mathrm{CH}\right), 5.92\left(1 \mathrm{H}, \mathrm{dd},{ }^{3} J_{\mathrm{PH}}=5.9 \mathrm{~Hz}\right.$, $\left.{ }^{3} J_{\mathrm{HH}}=11.5 \mathrm{~Hz}, \mathrm{P}-\mathrm{CH}-\mathrm{CH}\right), 7.02-7.86\left(14 \mathrm{H}, \mathrm{m}, \mathrm{H}_{\mathrm{Ar}}\right) .{ }^{13} \mathrm{C} \mathrm{NMR}\left(125.8 \mathrm{MHz}, \delta, \mathrm{CDCl}_{3}\right): 13.96$ and $13.99\left(2 \mathrm{~s}, 2 \mathrm{CH}_{3}\right) 45.34\left(\mathrm{~d},{ }^{1} J_{\mathrm{CP}}=134.6 \mathrm{~Hz}, \mathrm{P}-\mathrm{CH}\right), 49.45\left(\mathrm{~d},{ }^{2} J_{\mathrm{CP}}=4.6 \mathrm{~Hz}, \mathrm{P}-\mathrm{C}-\mathrm{CH}\right), 62.40$ and $62.82\left(2 \mathrm{~s}, 2 \mathrm{CH}_{2}\right), 123.78,123.59,131.9,134.27,134.31,167.54,167.01(\mathrm{~s}, \mathrm{C}$ of $\left.\mathrm{C}_{8} \mathrm{H}_{5} \mathrm{NO}_{2}\right) 120.33,120.49$ (2d, $\mathrm{C}_{\text {ortho }}$ ), 125.47 and 125.40 (d, $\mathrm{C}_{\text {para }}$ ), 129.66 (s, $\mathrm{C}_{\text {meta }}$ ), 149.59 (d, $\left.{ }^{2} J_{\mathrm{CP}}=9.6 \mathrm{~Hz}, \mathrm{C}_{\text {ipso }}\right), 150.1\left(\mathrm{~d},{ }^{2} J_{\mathrm{CP}}=8.4 \mathrm{~Hz}, \mathrm{C}_{\mathrm{ipso}}\right), 166.08\left(\mathrm{~d},{ }^{2} J_{\mathrm{CP}}=7.4 \mathrm{~Hz}, \mathrm{P}-\mathrm{C}-\mathrm{C}=\mathrm{O}\right), 167.77(\mathrm{~d}$, $\left.{ }^{3} J_{\mathrm{CP}}=18.7 \mathrm{~Hz}, \mathrm{P}-\mathrm{C}-\mathrm{C}-\mathrm{C}=\mathrm{O}\right)$. Anal. Calcd for $\mathrm{C}_{28} \mathrm{H}_{26} \mathrm{NO}_{9} \mathrm{P}$ (551): C, 60.98; H, 4.72; N, 2.54\%, Found: C, 60.95; H, 4.77; N, 2.48\%.

$\left(2 R^{*}, \quad 3 S^{*}\right)$-Dimethyl-2-(diphenoxyphosphoryl)-3-(1,2-dihydro-1 $\lambda^{6}$-benzol[d] isothiazol-3one-2-yl)-succinate (3c). White powder, yield 95\%, mp=132-134 ${ }^{\circ} \mathrm{C}$. IR (KBr) $\left(v_{\max }, \mathrm{cm}^{-1}\right): 1771$ $(\mathrm{C}=\mathrm{O}), 1716$ and $1736\left(\mathrm{CO}_{2} \mathrm{CH}_{3}\right), 1288(\mathrm{P}=\mathrm{O}) . \mathrm{MS},(\mathrm{m} / \mathrm{z}, \%): 432\left(\mathrm{M}-\mathrm{PhOH}\right.$ and $\left.\mathrm{CH}_{3} \mathrm{OH}, 2\right)$, $283\left(\mathrm{M}-\mathrm{C}_{7} \mathrm{H}_{4} \mathrm{NO}_{3}\right.$ and $\left.\mathrm{PhO}, 30\right), 223$ (283- $\left.\mathrm{CH}_{3} \mathrm{COOH}, 86\right), 165$ (283- $\left.-\mathrm{CO}_{2} \mathrm{CH}_{3}, 11\right), 94(\mathrm{PhOH}$, 26). ${ }^{31} \mathrm{P}$ NMR (202.4 MHz, $\left.\delta, \mathrm{CDCl}_{3}\right): 10.42\left[\mathrm{~s},\left(\mathrm{PhO}_{2} \mathrm{P}(=\mathrm{O})\right] .{ }^{1} \mathrm{H}\right.$ NMR $\left(300.1 \mathrm{MHz}, \delta, \mathrm{CDCl}_{3}\right)$ : 3.66 and $3.82\left(6 \mathrm{H}, 2 \mathrm{~s}, 2 \mathrm{CO}_{2} \mathrm{CH}_{3}\right), 4.52\left(1 \mathrm{H}, \mathrm{dd},{ }^{2} J_{\mathrm{PH}}=20.9 \mathrm{~Hz},{ }^{3} J_{\mathrm{HH}}=11.5 \mathrm{~Hz}, \mathrm{P}-\mathrm{CH}\right), 5.87(1 \mathrm{H}$, $\left.\mathrm{dd},{ }^{3} J_{\mathrm{PH}}=5.4 \mathrm{~Hz},{ }^{3} J_{\mathrm{HH}}=11.5 \mathrm{~Hz}, \mathrm{P}-\mathrm{CH}-\mathrm{CH}\right), 6.93-7.80\left(14 \mathrm{H}, \mathrm{m}, \mathrm{H}_{\mathrm{Ar}}\right) .{ }^{13} \mathrm{C} \mathrm{NMR}(75.4 \mathrm{MHz}, \delta$, $\left.\mathrm{CDCl}_{3}\right): 45.07\left(\mathrm{~d},{ }^{1} J_{\mathrm{CP}}=134.4 \mathrm{~Hz}, \mathrm{P}-\mathrm{CH}\right), 49.28\left(\mathrm{~d},{ }^{2} J_{\mathrm{CP}}=4.7 \mathrm{~Hz}, \mathrm{P}-\mathrm{C}-\mathrm{CH}\right), 53.32$ and 53.58 (s, $\left.2 \mathrm{OCH}_{3}\right), 123.85,131.81,134.36,166.97$ (s, $\mathrm{C}$ of $\left.\mathrm{C}_{7} \mathrm{H}_{5} \mathrm{NO}_{3} \mathrm{~S}\right) 120.31,120.45$ (2d, $\mathrm{C}_{\text {ortho }}$ ), 125.49 (d, $\left.\mathrm{C}_{\text {para }}\right), 129.7$ (s, $\left.\mathrm{C}_{\text {meta }}\right), 149.49$ (d, $\left.{ }^{2} J_{\mathrm{CP}}=9.8 \mathrm{~Hz}, \mathrm{C}_{\text {ipso }}\right), 149.97\left(\mathrm{~d},{ }^{2} J_{\mathrm{CP}}=8.6 \mathrm{~Hz}, \mathrm{C}_{\mathrm{ipso}}\right), 166.66$ (d, $\left.{ }^{2} J_{\mathrm{CP}}=7.5 \mathrm{~Hz}, \quad \mathrm{P}-\mathrm{C}-\mathrm{C}=\mathrm{O}\right), 168.97$ (d, $\left.{ }^{3} J_{\mathrm{CP}}=19.5 \mathrm{~Hz}, \mathrm{P}-\mathrm{C}-\mathrm{C}-\mathrm{C}=\mathrm{O}\right)$. Anal. Calcd for $\mathrm{C}_{25} \mathrm{H}_{22} \mathrm{NO}_{10} \mathrm{PS}$ (559): C, 53.66; H, 3.93; N, 2.50\%, Found: C, 53.64; H, 3.87; N, 2.45\%.

(2R* $\left.{ }^{*}, 3 S^{*}\right)$-Diethyl-2-(diphenoxyphosphoryl)-3-(1,2-dihydro-1 $\lambda^{6}$-benzol[d] isothiazol-3one-2yl)-succinate (3d). White powder, yield $92 \%, \mathrm{mp}=109-111^{\circ} \mathrm{C} . \mathrm{IR}(\mathrm{KBr})\left(v_{\max }, \mathrm{cm}^{-1}\right): 1771$ $(\mathrm{C}=\mathrm{O}), 1713$ and $1733\left(\mathrm{CO}_{2} \mathrm{CH}_{2} \mathrm{CH}_{3}\right), 1285(\mathrm{P}=\mathrm{O}) .{ }^{31} \mathrm{P} \mathrm{NMR}\left(202.4 \mathrm{MHz}, \delta, \mathrm{CDCl}_{3}\right): 10.73$ [s, $\left(\mathrm{PhO}_{2} \mathrm{P}(=\mathrm{O})\right] .{ }^{1} \mathrm{H}$ NMR $\left(300.1 \mathrm{MHz}, \delta, \mathrm{CDCl}_{3}\right)$ : 1.21 and $1.35\left(6 \mathrm{H}, 2 \mathrm{t}, \mathrm{CH}_{3}\right), 4.17-4.4(4 \mathrm{H}, \mathrm{m}$, $\left.\mathrm{OCH}_{2}\right), 4.58\left(1 \mathrm{H}, \mathrm{dd},{ }^{2} J_{\mathrm{PH}}=20.9 \mathrm{~Hz},{ }^{3} J_{\mathrm{HH}}=11.5 \mathrm{~Hz}, \mathrm{P}-\mathrm{CH}\right), 5.93\left(1 \mathrm{H}, \mathrm{dd},{ }^{3} J_{\mathrm{PH}}=5.4 \mathrm{~Hz},{ }^{3} J_{\mathrm{HH}}=11.5\right.$ $\mathrm{Hz}, \mathrm{P}-\mathrm{CH}-\mathrm{CH}), 7-7.9\left(14 \mathrm{H}, \mathrm{m}, \mathrm{H}_{\mathrm{Ar}}\right) .{ }^{13} \mathrm{C} \mathrm{NMR}\left(125.8 \mathrm{MHz}, \delta, \mathrm{CDCl}_{3}\right): 13.97$ and $14\left(2 \mathrm{~s}, 2 \mathrm{CH}_{3}\right)$ $45.27\left(\mathrm{~d},{ }^{1} J_{\mathrm{CP}}=133.7 \mathrm{~Hz}, \mathrm{P}-\mathrm{CH}\right), 49.40\left(\mathrm{~d},{ }^{2} J_{\mathrm{CP}}=4.6 \mathrm{~Hz}, \mathrm{P}-\mathrm{C}-\mathrm{CH}\right), 62.42$ and 62.85 (2s, $\left.2 \mathrm{CH}_{2}\right), 123.81,131.84,134.29,167.02\left(\mathrm{~s}, \mathrm{C}\right.$ of $\left.\mathrm{C}_{7} \mathrm{H}_{5} \mathrm{NO}_{3} \mathrm{~S}\right) 120.34,120.50$ (2d, $\left.\mathrm{C}_{\text {ortho}}\right), 125.42$ 
(d, $\mathrm{C}_{\text {para }}$ ), 129.68 (s, $\left.\mathrm{C}_{\text {meta }}\right), 149.58$ and 149.98 (d, $\mathrm{C}_{\mathrm{ipso}}$ ), 166.09 (d, $\left.{ }^{2} J_{\mathrm{CP}}=7.3 \mathrm{~Hz}, \mathrm{P}-\mathrm{C}-\mathrm{C}=\mathrm{O}\right)$, 167.02(d, ${ }^{3} J_{\mathrm{CP}}=19.4 \mathrm{~Hz}, \mathrm{P}-\mathrm{C}-\mathrm{C}-\mathrm{C}=\mathrm{O}$ ). Anal. Calcd for $\mathrm{C}_{27} \mathrm{H}_{26} \mathrm{NO}_{10} \mathrm{PS}$ (587): C, 55.19; H, 4.43; N, 2.38\%, Found: C, 55.03; H, 4.48; N, 2.29\%.

\section{Acknowledgements}

We gratefully acknowledge financial support from the Research Council of University of Sistan and Balouchestan.

\section{References}

1. (a) Yavari, I.; Islami, M. R.; Bijanzadeh, H. R. Tetrahedron 1999, 55, 5547. (b) Yavari, I.; Ramazani, A. Phosphorus, Sulphur, and Silicon 1997, 130, 73. (c) Yavari, I.; AnariAbbasinejad, M.; Hossaini, Z. Org. Biomol. Chem., 2003, 1, 560.

2. (a) Maghsoodlou, M. T.; Habibi-Khorassani, S. M.; Rofouei, M. K.; Adhamdoust, S. R.; Nassiri, M. Arkivoc 2006, (xii), 138. (b) Kalantari, M.; Islami, M. R.; Hasani, Z.; Saidi, K. Arkivoc 2006, (x), 55. (c) Islami, M. R.; Mollazehi, F.; Sheibani, H. Arkivoc 2005, (xy), 25. (d) Hassani, Z.; Islami, M. R.; Sheibani, H.; Kalantari, M.; Saidi, K. Arkivoc 2006, (i), 89.

3. 3. (a) Castelot-Deliencourt, G.; Pannecoucke, X.; Quirion, J-C. Tetrahedron Lett. 2001, 42, 1025; (b) Cohen, R. J.; Fox, D. L.; Eubank, J. F.; Salvatore, R. N. Tetrahedron Lett. 2003, 44, 8617. (c) Amiguse, E. J.; Migaud, M.E.; Tetrahedron Lett. 2004, 45, 1001. (d) Rassukana, Y. V.; Onysko, P. P.; Davydova, K. O.; Sinitsa, A. D. Tetrahedron Lett. 2004, 45, 3899. (e) Onysko, P. P.; Suvavola, O. A,; Rassukana, Y. V.; Chudakova, T. I.; Sinitsa, A. D. Tetrahedron Lett. 2003, 44, 1855. (f) Moravcova, J.; Heissigerova, H.; Kocalka, P.; Imberty, A.; Sykora, D.; Fris, M. Tetrahedron Lett. 2003, 44, 8797.

4. (a) Lefebver, I. M.; Evans Jr., S. A. J. Org. Chem. 1997, 62, 7532. (b) Kim S. K.; Hurh , E.Y.; Youn, J. N.; Park, J. I. J. Org. Chem. 1999, 64, 9272. (c) Joly, D. G.; Jacobsen, E. N. J. Am. Chem. Soc. 2004, 126, 4102. (d) Segall, Y.; Quistand, G. B.; Sparks, S. E.; Casida, J. E. Chem. Res. Toxicol. 2003, 16, 350.

5. (a) Corbridge, D. E. C. Phosphorus; Elsevier: Amsterdam, 1990; Chap. 13, pp 879-954. (b) Quin, D. L. A Guide to Organophosphorus Chemistry; John Wiley \& Sons: New York, 2000. (c) Walker, B. J. Organophosphorus Chemistry, Penguin Education, 1972. (d) Goodman and Gilman, A.; Hardman, J. G.; Limbird, L. E. The Pharmacological Basis of Therapeutics, 10 Edn., Mc. Graw-Hill: New York, 2001. (e) Gallant. J. E.; Pham. P. A. Expert Rev. Anti-infect. Ther. 2003, 1, 415.

6. Moonen, K.; Meenen, E. V.; Verwee, A.; Stevens, C. V. Angew. Chem. Int. Ed. 2005,44, 7407.

7. Moonen, K.; Laureyen, I.; Stevens, C. V. Chem. Rev. 2004, 104, 6177. 
8. Krawczyk, H.; Wolf, W. M.; Sliwinski, M. J. Chem. Soc., Perkin Trans.1 2002, 2794.

9. Brel, V. K. Synthesis. 2002, 1829.

10. Vanderhoydonck, B.; Stevens, C. V. Synthesis. 2004, 722.

11. (a) Breitmaier, E.; Voelter, W. Carbon-13NMR Spectroscopy; $3^{\text {rd }}$ Edn., VCH Publishers: New York,1990,pp 247-254. (b) Organic Compounds; Mander, L. N. Stereochemistry of Organic Compounds; John Wiley \& Sons: New York, 1994. (c) Karpus, M. J. Am. Chem. Soc., 1963, 85, 2870.

12. Breitmaier, E.; Voelter, E. Carbon-13 NMR Spectroscopy, VCH: New York, 1990, $3^{\text {rd }}$ Edn., p 250. 